\title{
Digital Light Synthesis 3D Printer
}

\author{
Mustafizur Rahman', Monjurul Islam Sabuj², MD Nahiduzzaman ${ }^{3}$ \\ Student, Mechanical Engineering, CUMT, Xuzhou, China, 1,2 \\ Student, Mechanical and Electrical Engineering, Hohai University, ${ }^{3}$
}

\begin{abstract}
D Printing technology, also known as Additive Manufacturing (AM), refers to processes used to generate a $3 \mathrm{D}$ object in which layers of material are successively formed under a computer controlled program to create a physical object. The 3D file source is usually sliced into several layers, each layer generating a set of computer controlled instructions. Both 3D printing and additive manufacturing reflect that the technologies share the theme of sequentiallayer material addition or joining throughout a 3D work. Yet, the technology used by most 3D printers since the 2010's, especially by hobbyist and consumer-oriented products, is Fused Filament Fabrication (FFF), also known as material extrusion or the proprietary Stratasys denomination's Fused Deposition Modeling (FDM). FDM was patented in 1989 by S. Scott Crump just before he launched the Stratasys company with his wife, Lisa Crump.
\end{abstract}

Keywords: Additive Manufacturing, 3D printers, Resin curing, DLP, Stereolithography, SLA.

\section{I.INTRODUCTION}

Additive manufacturing invention can be traced back to the 1980's by Japanese, French and American researchers. The very first patent of 3D printing was coined in 1984 by Chuck Hull of 3D Systems Corporation. Hull defined the 3D printing process as a system for generating three-dimensional objects by creating a cross-sectional pattern of the object to be formed. His invention consists of a stereolithography fabrication system, in which layers are added by curing photopolymers with ultraviolet light lasers. Stereolithography is still one very popular 3D printing manufacturing technique, also known as SLA. The term 3D printing originally referred to a process that deposited a binder material onto a powder bed with inkjet printer heads layer by layer. More recently, the term 3D printing is being used in popular vernacular to encompass a wider variety of additive manufacturing techniques. For professionals, the additive manufacturing name remains more popular for its broader sense and longer existence. Other terms are also employed, such as desktop manufacturing, rapid manufacturing, direct digital manufacturing, and rapid prototyping. 3D Printing of paper offers a full-color spectrum for designers that want to produce a realistic 3D model prior to advancing a product onto final engineering. Concrete is currently used for portions of housing, and even entire homes, in China. Chocolate, pizza, and cake decorations are being tested and employed for 3D Printing in the food sector. Also, the wide range of materials makes it possible to produce yarn with 3D Printing.

\section{STEREOLITHOGRAPHY SLA}

Stereolithography is a 3D Printing process which uses a computer-controlled moving laser beam, pre-programmed using CAM/CAD software. Stereolithography (SLA) is an industrial 3D printing process used to create concept models, cosmetic rapid prototypes, and complex parts with intricate geometries in as fast as 1 day. Stereolithography parts can be produced in a wide selection of materials, extremely high feature resolutions, and quality surface finishes are possible with SLA. The SL machine begins the 3D printing process by drawing the layers of the support structures, followed by the part itself, with an ultraviolet laser aimed onto the surface of a liquid thermoset resin. After a layer is imaged on the resin surface, the build platform shifts down and a recoating bar moves across the platform to apply the next layer of resin. The process is repeated layer by layer until the build is complete. Newly built parts are taken out of machine and into a lab where solvents are used to remove any additional resins. When the parts are completely clean, the support structures are manually removed. From there, parts undergo a UV-curing cycle to fully solidify the outer surface of the part. The final step in the SL process is the application of any custom or customer-specified finishing. Parts built in SL should be used with minimal UV and humidity exposure so they don't degrade. 


\section{International Advanced Research Journal in Science, Engineering and Technology}

Vol. 8, Issue 7, July 2021

DOI: 10.17148/IARJSET.2021.8702

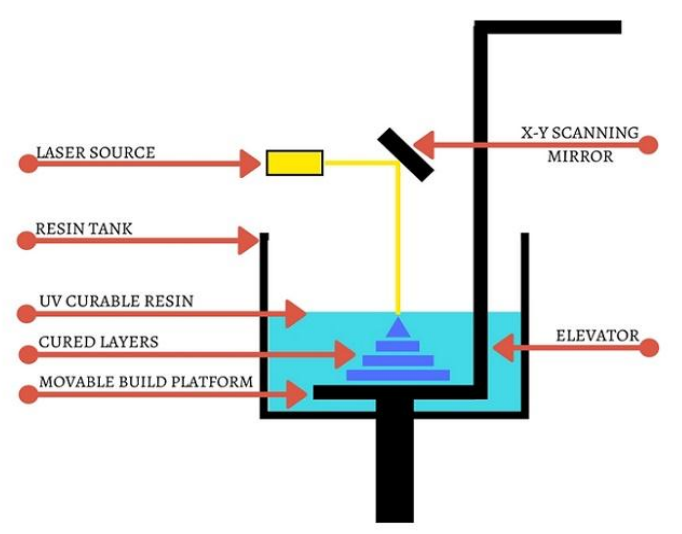

Stereolithography is good for producing accurate prototypes and models. Stereolithography is well used for creating accurate 3D models of anatomical regions of a patient, used to aid in diagnosis and for pre-planning and implant design and manufacture. It is also good for use in concept models and scale models. Stereolithography is used for prototyping in order to assess design and for part validation. This is due to accuracy and its ability to produce irregular shapes.

Unlike older generations of SL, today's machines offer a range of thermoplastic-like materials to choose from, with several variants to mimic polypropylene, ABS, and glass-filled polycarbonate available.

- Polypropylene: A flexible, durable resin that mimics a stiff polypropylene. It can withstand harsh mechanical treatment and is great for fine details - sharp corners, thin walls, small holes, etc.

- Polypropylene/ABS blend: Strong, white plastic similar to a CNC machined polypropylene/ABS blend. It works well for snap fits, assemblies, and demanding applications.

- ABS: Variations of ABS mimics include a clear, low-viscosity resin that can be finished clear; an opaque black plastic that blocks nearly all visible light, even in thin sections; a clear, colourless, water-resistant plastic good for lenses and flow-visualisation models; and a micro-resolution resin that enables production of parts with extremely fine features and tight tolerances.

- Polycarbonate: A ceramic-filled PC material that provides strength, stiffness, and temperature resistance, but can be brittle.

\section{III.Digital Light Processing DLP}

DLP stands for digital light processing, and is a type of vat polymerization. Vat polymerization 3D printing technologies make use of a (liquid) photopolymer resin which is able to cure (solidify) under a light source. In the world of vat polymerization, there are two main technologies: SLA and DLP. Naturally, both use resin and a light source to produce parts, the main difference being the type of light source which is used to cure the resin. With a DLP 3D printer, the light source is a specially developed digital light projector screen. Thanks to this screen, DLP is generally considered to be faster than SLA, and here's why: With SLA, the laser has to individually cure the resin in a "point to point" technique. On the other hand, a DLP projector screen flashes an image of a layer all at once! Thus all points of a layer can be cured simultaneously. In this way, the print speed is increased in comparison to SLA since it takes less time to cure a single layer. Since the DLP is a digital technology, the 2D image that is projected is composed of pixels. When translated into three dimensions, they become voxels. The light source of a DLP 3D printer itself, an LED screen, means nothing without a digital micromirror device (DMD), the "heart" of every DLP chipset. A DMD contains hundreds of thousands or even millions of small micromirrors that direct the light and create the pattern of a layer onto the bottom of the resin tank. The resolution of a printed part using a DLP 3D printer usually corresponds to the number of micromirrors inside a DMD device. 


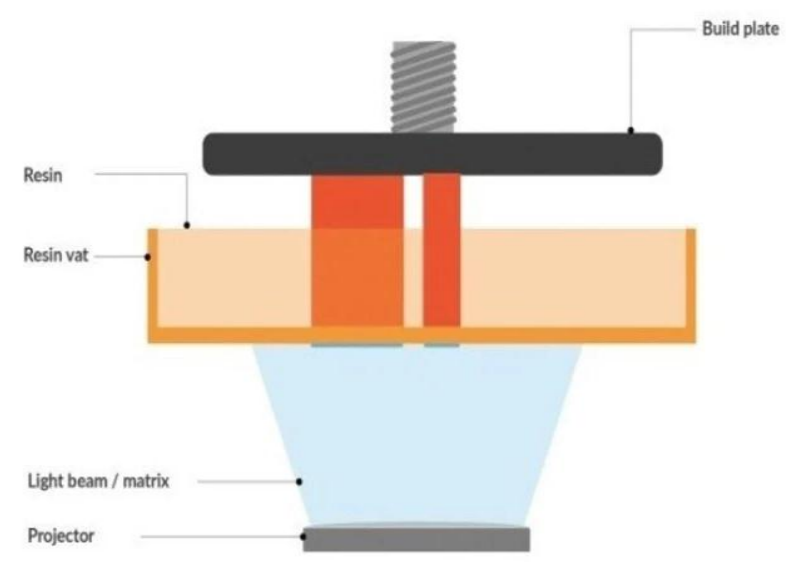

SLA vs DLP

Since a DLP 3D printer uses a digital projector screen, the resolution of your print directly corresponds to the resolution of your projector. In other words, a cheaper projector will likely mean reduced resolution This is in contrast to SLA, where even cheaper devices are likely to have decent resolution (i.e. a narrow beam width). Another limitation of DLP printers is the boxy surface finish. Since voxels are rectangular, curved sections of a print tend not have a very smooth finish when compared to SLA. The good thing is that the problem with voxels and curves can be solved by sanding the part after printing.

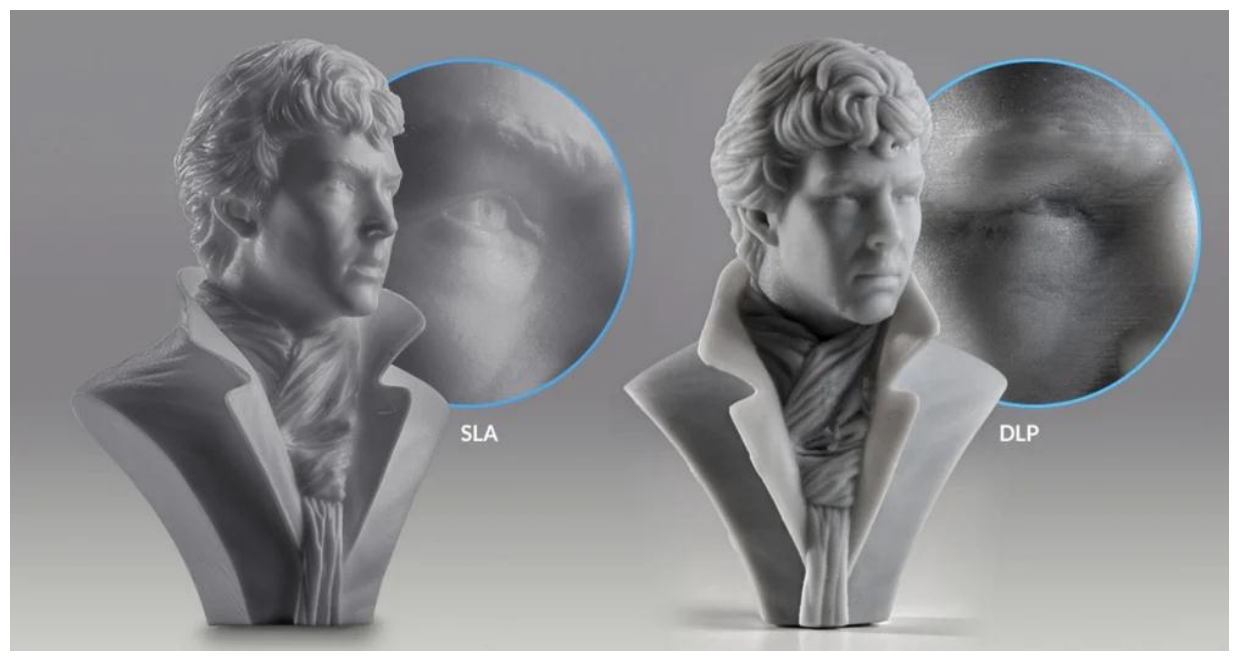

Both DLP and SLA printers use resins, so the cost of printing should be similar. However, keep in mind that cost greatly depends on the manufacturer of the resin or even the printer.

- The primary difference between DLP and SLA is the light source; SLA uses a UV laser beam while the DLP uses UV light from a projector.

- In DLP, the UV light source remains stationary and it cures the complete layer of resin at a time. In SLA, the laser beam moves from point to point tracing the geometry.

- Since the curing (hardening) of the resin is done from point to point, SLA 3D printing is more accurate and the quality of the print is also better in comparison to DLP 3D printing.

- The DLP 3D printer works faster and cuts down the 3D printing time significantly.

- In DLP 3D printer, the operator can control the intensity of UV light source and thereby control its effect on the resin. In SLA, the intensity of the laser beam cannot be adjusted and you have to change the laser light completely for different resin effect. 
- The UV light source in DLP is less expensive and can be easily replaced. Laser lights are expensive in comparison.

- DLP is more suitable to build one-off small and intricate part while SLA is more suitable for printing several intricate parts in one go.

- DLP is useful in quickly printing large part without much detail while SLA is perfect to print a large part with intricate details.

DLP



SLA

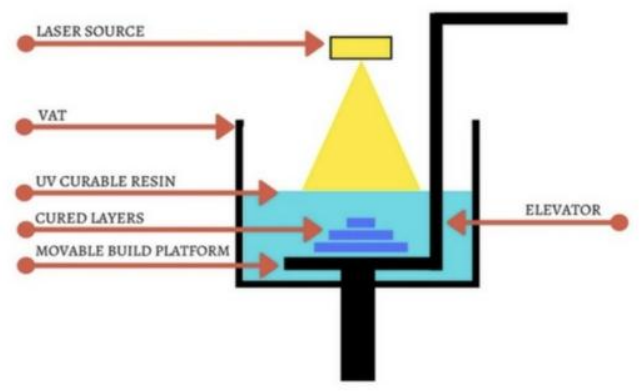

Above: A diagrammatic representation of the working of DLP and SLA printing processes

Resolution shows up more often than any other value in 3D printer spec sheets, but it's also a common ground for confusion. The basic units of the SLA and DLP processes are different shapes, making it difficult to compare the different machines by numerical specifications alone. In 3D printing, there are three dimensions to consider: the two planar 2D dimensions ( $\mathrm{X}$ and $\mathrm{Y}$ ) and the third vertical $\mathrm{Z}$ dimension that makes 3D printing.

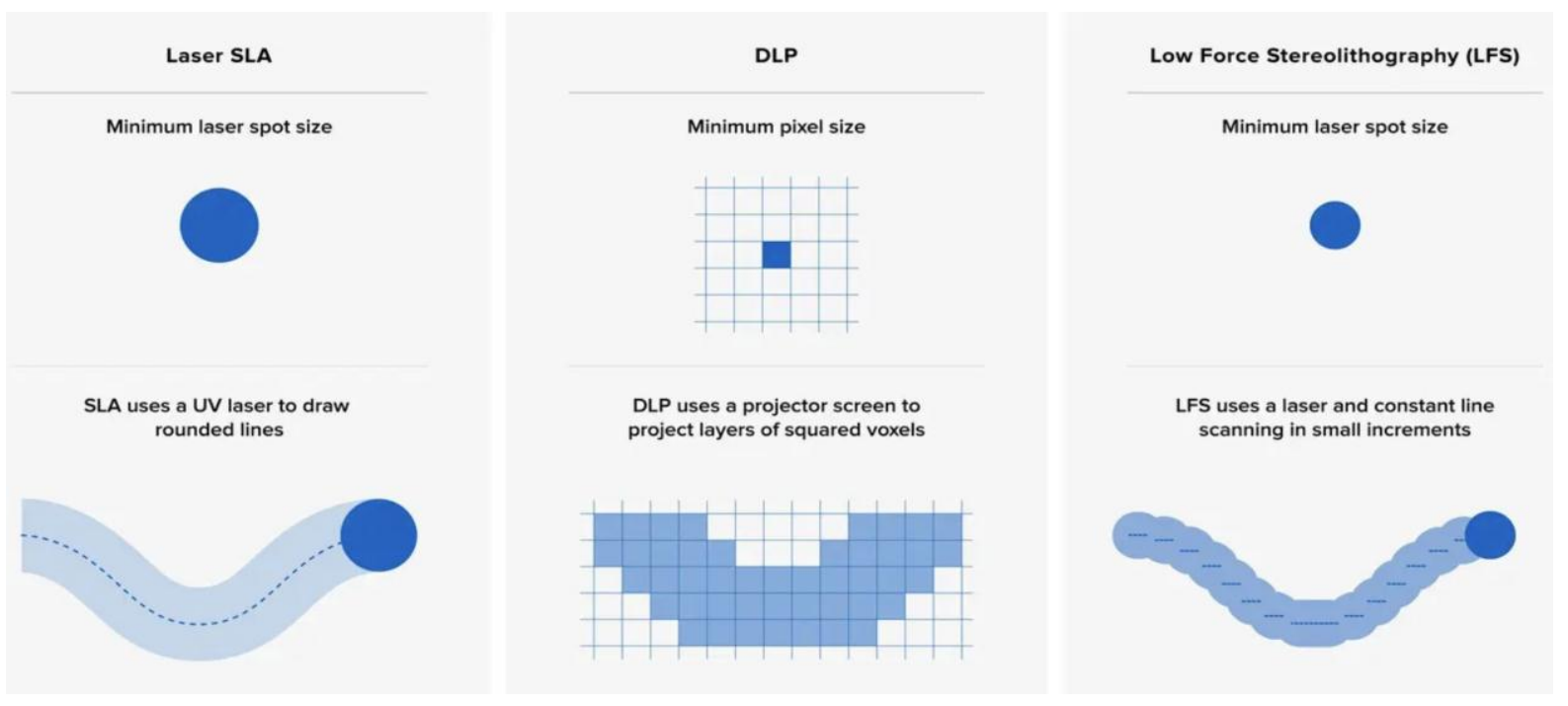

$\mathrm{Z}$ resolution is defined by the layer thicknesses a 3D printer can produce. Resin 3D printers like SLA and DLP offer some of the finest $\mathrm{Z}$ resolutions - thinnest layers - of all 3D printing processes and users can normally choose from a range of layer height options between 25-300 microns, allowing designers to strike a balance between detail and speed. 
In DLP 3D printing, XY resolution is defined by the pixel size, the smallest feature the projector can reproduce within a single layer. This depends on the resolution of the projector, the most common being full HD (1080p), and its distance from the optical window. As a result, most desktop DLP 3D printers have a fixed XY resolution, generally between 35 to 100 microns.

For SLA 3D printers, XY resolution is a combination of the laser's spot size and the increments by which the laser beam can be controlled. For example, the Form 3 LFS 3D printer features a laser with an 85 micron spot size, but because of the constant line scanning process, the laser can move in smaller increments and the printer can consistently deliver parts with 25 micron XY resolution.

However, resolution in itself is often just a vanity metric. It offers some indication, but it doesn't necessarily correlate directly with accuracy, precision, and print quality.

\section{Accuracy and Precision}

Because 3D printing is an additive process, each layer introduces an opportunity for inaccuracy, and the process by which layers are formed affects the level of precision, defined as the repeatability of the accuracy of each layer. Accuracy and precision depend on many different factors: 3D printing process, materials, software settings, post-processing, and more. In general, both SLA and DLP resin 3D printers are among the most accurate and precise 3D printing processes. Differences in accuracy and precision are often better explained by the differences between machines by various manufacturers than differences between the technologies themselves.

For example, entry-level SLA or DLP printers might use off-the-shelf projectors, lasers, or galvanometers, and their manufacturers will try to get the best performance possible out of these parts.

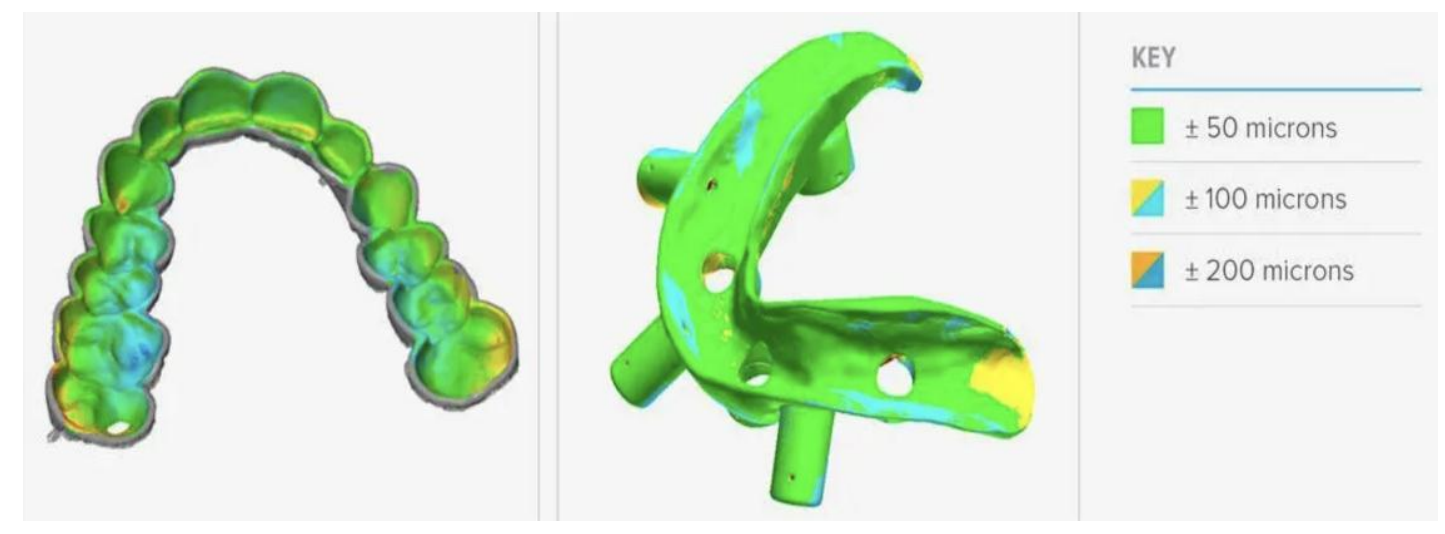

Accuracy Accuracy and precision are crucial for parts like dental splints (left) and surgical guides (right). Calibration is also crucial. With DLP projectors, manufacturers need to deal with the non-uniform light distribution on the build plane and optical distortion of the lenses - meaning that pixels in the middle are not the same size or shape as pixels on the edges. SLA 3D printers use the same light source for every part of the print, which means it's uniform by definition, but they still require extensive calibration to account for distortions. Even a 3D printer with the highest quality components and degree of calibration can produce widely varying results depending on the material. Different resins require optimized material settings to perform as intended, which might not be available for off-the-shelf materials or resins that are not thoroughly tested with a specific 3D printer model.

\section{Build Volume}

With DLP 3D printers, there's a direct trade-off between resolution and build volume. The resolution depends on the projector, which defines the number of pixels/voxels available. If one moves the projector closer to the optical window, the pixels get smaller, which increases the resolution, but limits the available build area. Some manufacturers stack multiple projectors next to each other or use a high-definition $4 \mathrm{~K}$ projector to increase the build volume, but this leads to substantially higher costs that often price these machines out of the desktop market. 


\section{International Advanced Research Journal in Science, Engineering and Technology}

Vol. 8, Issue 7, July 2021

DOI: $10.17148 /$ IARJSET.2021.8702

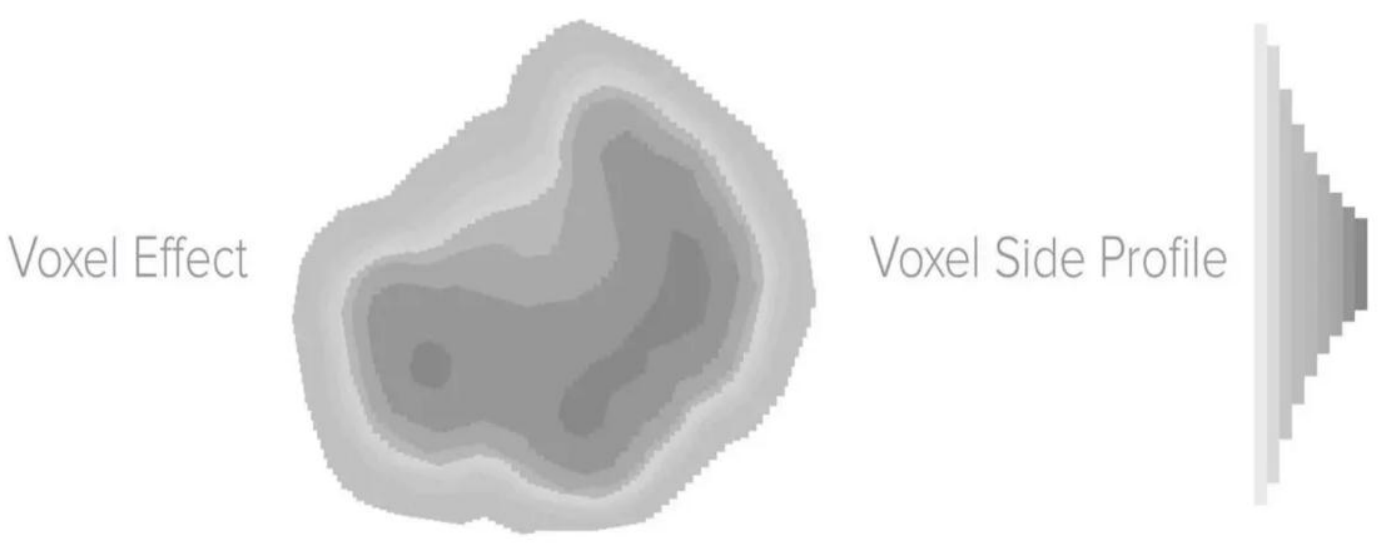

As a result, DLP 3D printers are generally optimized for specific use cases. Some have a smaller build volume and offer high resolution to produce small, detailed pieces like jewelry, while others can produce bigger parts but at a lower resolution.

The stereolithography process is inherently more scalable, since an SLA 3D printer's build volume is completely independent of the resolution of the print. A single print can be any size and any resolution at any location within the build area. This makes it possible to $3 \mathrm{D}$ print large parts at high resolution or a large batch of detailed small parts to increase throughput with the same machine.

The other main barrier to increasing build volume in both SLA and DLP 3D printers is the peel force. When printing larger parts, the forces exerted on the parts increase exponentially as a cured layer separates from the tank.

\section{Surface Finish}

LA and DLP resin 3D printers both known for creating parts with the smoothest surface finish of all 3D printing processes. When we describe the differences, in most cases these are only visible on tiny parts or highly detailed models. Because objects are made of layers in 3D printing, 3D prints often have visible, horizontal layer lines. However, because DLP renders images using rectangular voxels, there is also an effect of vertical voxel lines.

DLP 3D printers render images using rectangular voxels, which causes an effect of vertical voxel lines. In this image, see the vertical voxel lines as they appear naturally on the left, and then outlined to more easily identify on the right.

Because the unit is rectangular, voxels also have an effect on curved edges. Think of building a round shape out of LEGO bricks - the edges will appear stepped on both the $\mathrm{Z}$ axis and the $\mathrm{X}-\mathrm{Y}$ plane.

\section{Throughput}

Raw print speed for SLA and DLP resin 3D printers is comparable in general. As the projector exposes each entire layer all at once, print speed in DLP 3D printing is uniform and depends only on the height of the build, whereas, SLA 3D printers draw out each part with a laser. As a rule of thumb, this results in SLA 3D printers being comparable or faster when printing small or medium-size single parts, while DLP 3D printers are faster to print large, fully dense prints, or builds with multiple parts that fill up much of the platform. But once again, it's worth considering the trade-off between resolution and build volume for DLP printers. A small DLP 3D printer can print a small part or a (small) batch of smaller parts fast, at high resolution, but the build volume limits the part size and the throughput. A different machine with a larger build volume can print larger parts or a batch of smaller parts faster, but at a lower resolution than SLA. SLA 3D printers can produce all of these options in one machine and offer the user the freedom to decide whether she wants to optimize for resolution, speed, or throughput. Speed can also depend on the material choice. Draft Resin fast-printing resin that is ideal for initial prototypes, rapid iterations, as well as dental and orthodontic models. From fast print initiation speeds to minimal support removal, wash, and cure times, Draft Resin has an optimized workflow to truly maximize efficiency. 


\section{International Advanced Research Journal in Science, Engineering and Technology}

Vol. 8, Issue 7, July 2021

DOI: 10.17148/IARJSET.2021.8702
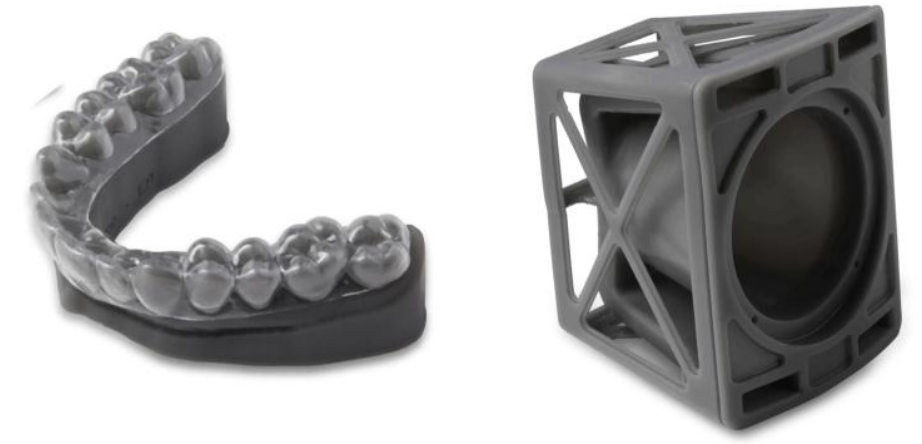

\begin{tabular}{|c|c|}
\hline $\begin{array}{l}\text { GREY RESIN } \\
100 \text { MICRONS }\end{array}$ & $\begin{array}{l}\text { DRAFT RESIN } \\
200 \text { MICRONS }\end{array}$ \\
\hline $71 \mathrm{~min}$ & $18 \mathrm{~min}$ \\
\hline
\end{tabular}

\begin{tabular}{|c|c|}
\hline $\begin{array}{l}\text { GREY RESIN } \\
100 \text { MICRONS }\end{array}$ & $\begin{array}{l}\text { DRAFT RESIN } \\
200 \text { MICRONS }\end{array}$ \\
\hline 21 hrs $46 \mathrm{~min}$ & $8 \mathrm{~h} 43 \mathrm{~min}$ \\
\hline
\end{tabular}

\section{REFERENCES}

1. J. M. Pearce, Science 337, 1303-1304 (2012).

2. H. Lipson, M. Kurman, Fabricated: The New World of 3D Printing (Wiley,

Indianapolis, 2013).

3. B. Derby, Science 338 , 921-926 (2012).

4. A. Atala, F. K. Kasper, A. G. Mikos, Sci. Transl. Med. 4 , 160rv12 (2012).

5. B. C. Gross, J. L. Erkal, S. Y. Lockwood, C. Chen, D. M. Spence, Anal. Chem. 86, 3240-3253 (2014).

6. K. Sun et al., Adv. Mater. 25 , 4539-4543 (2013).

7. G. Chisholm, P. J. Kitson, N. D. Kirkaldy, L. G. Bloor, L. Cronin, Energy Environ. Sci. 7, 3026-3032 (2014).

8. M. D. Symes et al., Nat. Chem. 4 , 349-354 (2012).

9. P. Chakraborty, R. N. Zuckermann, Proc. Natl. Acad. Sci. U.S.A. 110 , 13368-13373 (2013).

10. P. J. Kitson et al., Cryst. Growth Des. 14 , 2720-2724 (2014).

11. J. L. Erkal et al., Lab Chip 14, 2023-2032 (2014).

12. X. Zheng et al., Science 344, 1373-1377 (2014).

13. T. A. Schaedler et al., Science 334, 962-965 (2011).

14. J. Bauer, S. Hengsbach, I. Tesari, R. Schwaiger, O. Kraft, Proc. Natl. Acad. Sci. U.S.A. 111 , 2453-2458 (2014).

15. E. B. Duoss et al., Adv. Funct. Mater. 24 , 4905-4913 (2014).

16. I. Gibson, D. W. Rosen, B. Stucker, Additive Manufacturing Technologies: Rapid Prototyping to Direct Digital Manufacturing (Springer, New York, 2010).

17. S. C. Ligon, B. Husár, H. Wutzel, R. Holman, R. Liska, Chem. Rev. 114, 557-589 (2014).

18. Y. Yagci, S. Jockusch, N. J. Turro, Macromolecules 4 3, 6245-6260 (2010).

19. P. F. Jacobs, Rapid Prototyping \& Manufacturing: Fundamentals of StereoLithography (Society of Manufacturing Engineers, Dearborn, MI, 1992).

20. T. C. Merkel, I. Pinnau, R. Prabhakar, B. D. Freeman, Materials Science of Membranes for Gas and Vapor Separation (Wiley, West Sussex, UK, 2006), pp. 251-270.

21. D. Dendukuri et al., Macromolecules 41, 8547-8556 (2008).

22. D. Dendukuri, D. C. Pregibon, J. Collins, T. A. Hatton, P. S. Doyle, Nat. Mater. 5,365-369 (2006).

23. J. M. Gonzalez-Meijome, V. Compañ-Moreno, E. Riande, Ind. Eng. Chem. Res. 47,3619-3629 (2008).

24. J. A. Rogers, T. Someya, Y. Huang, Science 327, 1603-1607 (2010).

25. S. Bauer et al., Adv. Mater. 26, 149-161 (2014).

26. N. Travitzky et al., Adv. Eng. Mater. 16, 729-754 (2014).

27. C. Cvetkovic et al., Proc. Natl. Acad. Sci. U.S.A. 11 1 , 10125-10130 (2014).

28. Y. Lu, G. Mapili, G. Suhali, S. Chen, K. Roy, J. Biomed. Mater. Res. A 77, 396-405 (2006). 